\title{
Microscopic notes on the perinucleolar chromatin region in immature and mature human B-leukemia lymphocytes
}

\author{
K. SMETANA ${ }^{1, *}$, J. KARBAN ${ }^{2}$, I. JIRASKOVA ${ }^{1}, \mathrm{H}, \mathrm{KLAMOVA}^{1}$, M. TRNENY ${ }^{1,2}$ \\ ${ }^{1}$ Institute of Hematology and Blood Transfusion, Prague; ${ }^{2} 1^{\text {st }}$ Medical Faculty of Charles University, Prague, Czech Republic \\ ${ }^{*}$ Correspondence: karel.smetana@uhkt.cz
}

Received November 4, 2012 / Accepted December 5, 2012

\begin{abstract}
Perinucleolar region was studied in lymphocytes of patients suffering from chronic B lymphocytic leukemia to provide more information on the perinucleolar-condensed chromatin - heterochromatin - during the maturation of these cells. The perinucleolar heterochromatin of lymphocytes in smear preparations was visualized using a simple, but sensitive cytochemical method for the demonstration of DNA. The perinucleolar heterochromatin was also easily visible as unstained perinucleolar regions in specimens stained for RNA. In addition, the perinucleolar heterochromatin of lymphocytes was distinct and apparent in the transmission electron microscope using conventional as well as cytochemical methods for visualization of chromatin structures. Despite the great variability, the maturation of leukemic lymphocytes was accompanied by an increased width of the perinucleolar heterochromatin shell.

It seems to be also interesting that the perinucleolar region of both immature as well as mature leukemia lymphocytes contains heterochromatin bodies about $2 \mu \mathrm{m}$ in diameter. They appeared to be a regular component of the perinucleolar heterochromatin shell and were apparently different from other nuclear bodies present at the nucleolus. In contrary to other known nuclear bodies, perinucleolar heterochromatin bodies in leukemia lymphocytes consisted only of conglomerates of DNA containing chromatin fibrils and did not contain other structural components including RNA. The presence of perinucleolar heterochromatin bodies in the perinucleolar region of leukemia lymphocytes is not contradictory with the present knowledge on that nuclear territory. They might be associated with presumed special perinucleolar DNA loci, which according some previous studies were more expressed in malignant cells.
\end{abstract}

Key words: leukemic B-lymphocytes, perinucleolar heterochromatin

It seems to be clear that the perinucleolar region represents a very special nuclear compartment that is involved in the transcription of not-ribosomal RNA species and various cell states including the malignancy [1-4]. On the other hand, the knowledge of the morphology and organization of the perinucleolar DNA and chromatin is very limited [5]. The condensed perinucleolar heterochromatin is forming a discontinuous shell around the nucleolar body. It is generally known that its inner layer penetrates into the nucleolar body and forks and participates in the ribosomal RNA transcription [6] On the other hand; no adequate information exists on the size, i.e. width and organization of the condensed perinucleolar heterochromatin during the cell maturation.

The nucleated blood cells and especially leukemia lymphocytes of chronic lymphocytic leukemia represent a very convenient model for such study because they are present in the peripheral blood or bone marrow in a satisfactory number and their maturation stages including the nucleolar morphology and size are well known [7-9]. It is also generally known that mature lymphocytes are mostly in G0 or early G1 phase of the cell cycle. In addition, mature small lymphocytes with small ring-shaped nucleoli represent post-mitotic cells and mature small lymphocytes with micronucleoli and clumped condensed chromatin are in a pre-apoptotic state. In contrary, large lymphocytes with large nucleoli and a fine chromatin structure are immature and proliferating in the $\mathrm{S}$ phase and some of them in the pre-mitotic state. The nucleolar bodies in both immature and mature lymphocytes are easily seen in specimens stained for RNA and are surrounded by a distinct but unstained condensed heterochromatin shell. In addition, the visualization of the perinucleolar condensed chromatin - heterochromatin - in specimens stained for DNA in both light and electron microscope might provide complementary information of its morphology and organization. 


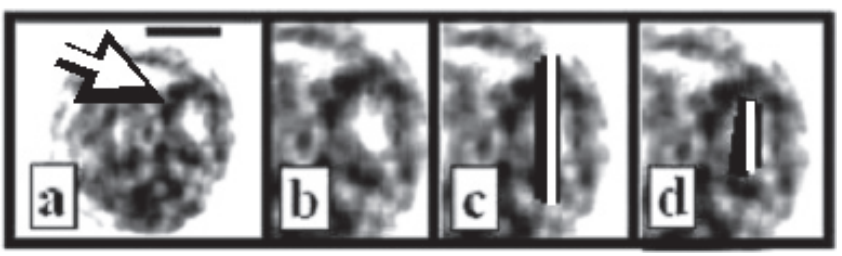

Figure 1. Mature lymphocyte stained for DNA. White pointer - nucleolus with a wide perinucleolar heterochromatin shell. The black bold bar represents $2 \mu \mathrm{m}$ (a). Image processing, larger magnification and increased contrast of the original captured image $(b-d)$. The magnified line represents the measured nucleolar body surrounded by the perinucleolar heterochromatin (c) and measured nucleolar body without the perinucleolar shell (d).

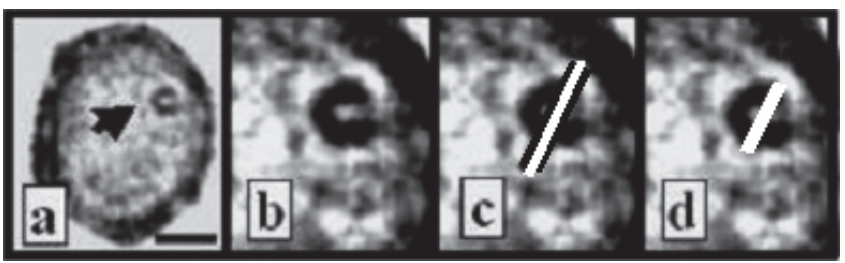

Figure 2. Mature lymphocyte stained for RNA. Black pointer - ring shaped nucleolus surrounded by a distinct unstained perinucleolar heterochromatin shell. For other legend see Fig. 1.

The results demonstrated that the width of the perinucleolar heterochromatin shell apparently increases during the maturation of lymphocytes in both untreated and treated patients with the cytostatic therapy despite a great variability. The perinucleolar heterochromatin bodies observed by both light as well as electron microscopy apparently were a regular part of the perinucleolar chromatin region. However, the increasing size the heterochromatin bodies in the perinucleolar region was not significant similarly as the perinucleolar heterochromatin DNA condensation state.

\section{Material and methods}

Perinucleolar condensed chromatin structures (heterochromatin) were studied in immature and mature lymphocytes of the peripheral blood of patients suffering from chronic lymphocytic leukemia without any respect to the state and stage of the disease using both light and electron microscopic approach. 3 patients were untreated and 3 patients were treated with the current cytostatic therapy (Fludara, Bayer- Schering, Germany) at the time of taking samples for the quantitative light microscopic cytochemical study. The median of the lymphocytic count was about 40 (untreated patients) and $150 \times 10^{9} / \mathrm{L}$ (treated patients) including approximately 15-20 per cent of immature cells. In addition, lymphocytes of 5 other untreated patients were studied using the electron microscopy including the DNA and RNA cytochemistry. Peripheral venous blood was origi-

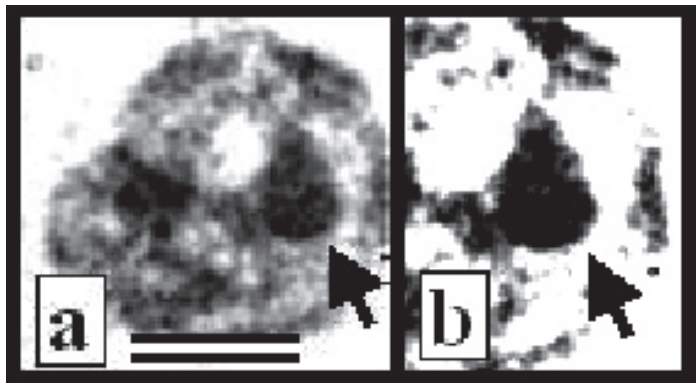

Figure 3. Immature lymphocyte with a large perinucleolar chromatin body (pointer) stained for DNA (a). The image processing slightly increased the size and contrast of the perinucleolar chromatin body (b). For other legend see Fig. 1.

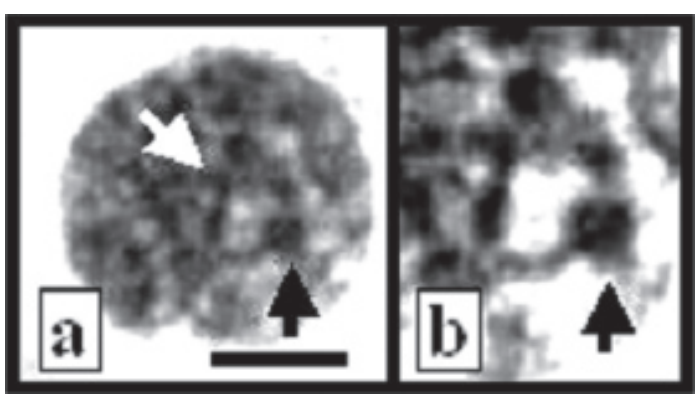

Figure 4. Mature lymphocyte with a distinct perinucleolar chromatin shell and perinucleolar chromatin body (a). Note that the perinucleolar body (black pointer) appears to be a part of the perinucleolar chromatin shell (white pointer). For other legend see Fig. 1 and 3.

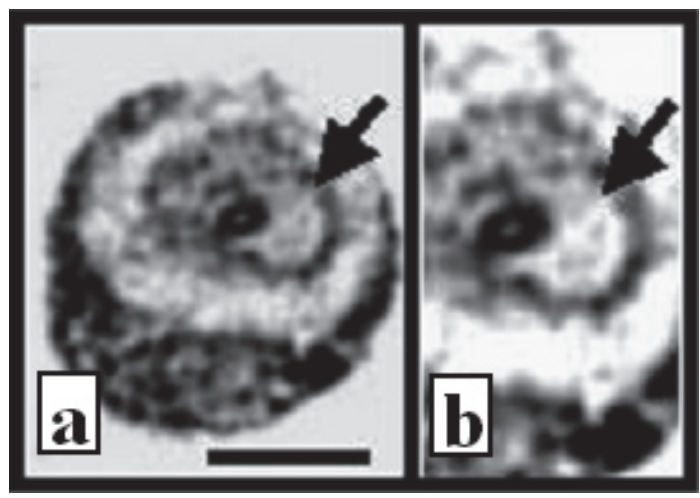

Figure 5. Pre-apoptotic mature lymphocyte with a characteristic chromatin condensation at the nuclear envelope and in the perinucleolar region (pointer). Note that chromatin does not exhibit a positive staining for RNA (a). The unstained chromatin regions are more apparent after image processing that increased the magnification and contrast of heterochromatin unstained regions (b). For other legend see Fig. 2.

nally taken for diagnostic purposes using current diagnostic procedures including the flow cytometry. The ethics committee of the Institute approved the protocols and methods used for the present study. 


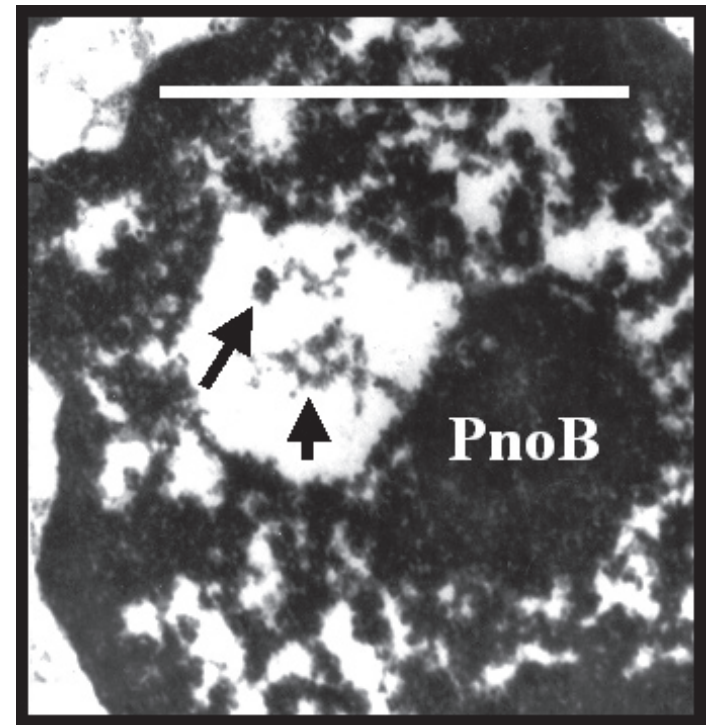

Figure 6. "Small" magnification of a ring shaped nucleolar body with intranucleolar cundensed chromatin (pointers) after complete extraction of RNA containing structures with pepsin and ribonuclease. Electron micrograph. The distinct perinucleolar heterochromatin body (PnoB) appears to be a part of the perinucleolar chromatin shell that is also connected with extranucleolar chromatin structures. The bold bar indicates $2 \mu \mathrm{m}$.

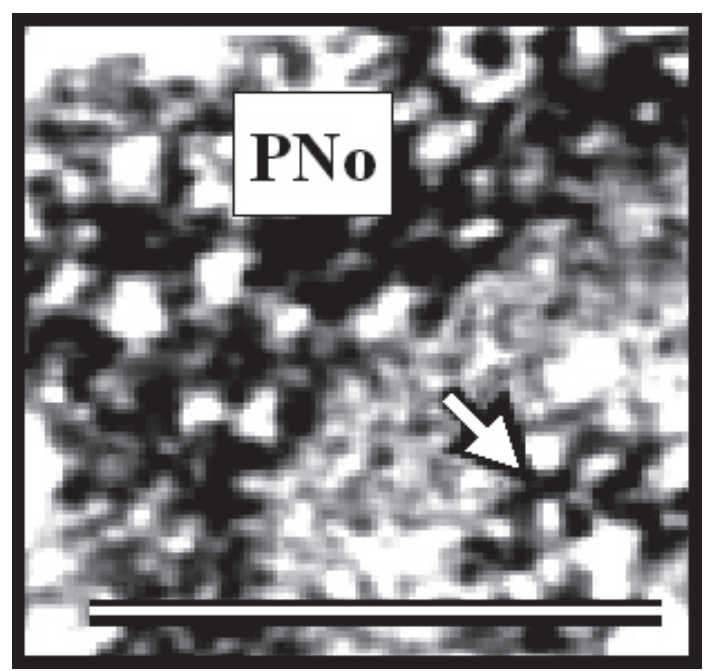

Fig. 7. Chromatin fibrils in the perinucleolar heterochromatin region (PNo) and within the nucleolar body (small pointers). The bold bar represents $200 \mathrm{~nm}$ (a). Chromatin fibrils are more apparent after increased magnification and contrast by image processing. For other legend see the previous Figure.

Light microscopy (Fig. 1-5). Perinucleolar heterochromatin in lymphocytes was visualized in methanol fixed dry peripheral blood smears by a simple sensitive method for demonstration of DNA using methylene blue buffered with
McIlvain's buffer to $\mathrm{pH} 5.3$ after hydrolysis with $1 \mathrm{~N} \mathrm{HCl} \mathrm{[10,}$ 11, see Fig. 1]. The unstained - negative distinct perinucleolar heterochromatin images in lymphocytes were also visualized in unfixed dry specimens stained for RNA with similarly prepared methylene blue solution at the same $\mathrm{pH}$, but without any previous treatment and fixation [12, 13, see Fig. 2].

Micrographs were captures with a Camedia digital camera C4040 ZOOM (Olympus, Japan) placed on Jenalumar microscope (Zeiss, Germany). The double adapter on the microscope increased the magnification of captured images transferred to the computer screen. The increased contrast by image processing (Fig. 1-5) facilitated easy measurements of the major axis length, i.e. largest diameter of measured structures [14, 15] using Quick Photoprogram (Olympus, Japan). Such measurements provided approximate, but useful information on the size of nucleolar or perinucleolar heterochromatin regions. The mean measurements of nucleolar bodies without as well as with the perinucleolar heterochromatin shell (see Fig. 1,2) and perinucleolar heterochromatin bodies in each of immature and mature lymphocytes were evaluated in all studied leukemic patients (see above). In addition, the perinucleolar heterochromatin optical density was measured in smeared lymphocytes stained for DNA after the conversion of captured blue signals to gray scale using the red channel of NIH Image Program, Scion for Windows (Scion Corp., USA). The condensed chromatin density reflecting its concentration in perinucleolar regions was expressed in arbitrary units calculated by subtracting the mean background density surrounding each measured cell from the condensed chromatin density measured in the perinucleolar region [8].

The results of all measurements at the single cell level such as mean, standard deviation and significance were evaluated using Primer of Biostatistic Program, version 1 developed by S.A. Glantz (McGraw-Hill, Canada, 1968).

Electron microscopy (Fig. 6-7). For the conventional electron microscopy, peripheral blood samples were fixed in glutaraldehyde and post-fixed in osmium tetroxide (not shown). For the selective chromatin visualization, peripheral blood samples were shortly fixed in formaldehyde and digested with pepsin followed by ribonucleases to remove all RNA containing structures (Fig. 6). Some specimens were also shortly digested with pepsin to provide better visibility of structures containing both RNA and especially DNA containing structures (Fig. 7). The digestion was terminated by a short treatment in ice-cold trichloracetic acid [for details see 11, 16]. Then all specimens were dehydrated in ethanol containing the decreasing concentration of uranyl acetate to prevent artificial dislocations of fine nuclear structures [16] and embedded in Epon (Serva, Germany) and Durcupan (Fluka, Switzerland) mixture prepared according to Mollenhauer [17]. In that mixture Durcupan replaced originally used Araldite. Ultrathin sections stained with uranyl acetate followed by lead citrate were examined with Philips 300 and Philips Morgagni electron microscopes (Philips, Netherland). Captured electron micrographs were processed to increase or decrease of the contrast 
Table 1. The perinucleolar heterochromatin width, perinucleolar body largest diameter (axis) and density in immature and mature lymphocytes stained for DNA (mean and standard deviation)*

\begin{tabular}{|c|c|c|c|c|c|c|}
\hline Lymphocytes & Th & $\begin{array}{c}\text { PeriNoCh } \\
\text { density }^{\bullet}\end{array}$ & $\mathrm{NoB}^{\wedge}$ & $\begin{array}{c}\text { NoB }+ \text { PeriNoCh } \\
\text { width }\end{array}$ & $\begin{array}{c}\text { PeriNoCh } \\
\text { width }^{\wedge}\end{array}$ & $\begin{array}{c}\text { PeriNo } \\
\text { ChB }^{\wedge}\end{array}$ \\
\hline immature & 0 & $86.3 \pm 9.7$ & $2.6 \pm 0.3$ & $4.8 \pm 0.2$ & $2,2 \pm 0.2$ & $1.9 \pm 0.3$ \\
\hline mature & & $94.5 \pm 9.6$ & $1.5 \pm 0.2^{\#}$ & $3.8 \pm 0.4^{\#}$ & $2.3 \pm 0.4$ & $2.2 \pm 0.4$ \\
\hline immature & + & $92.4 \pm 16.3$ & $2.8 \pm 0.1$ & $4.7 \pm 0.1$ & $1.9 \pm 0.1$ & $2.0 \pm 0.4$ \\
\hline mature & & $99.5 \pm 16.5$ & $1.7 \pm 0.1^{\#}$ & $3.9 \pm 0.5$ & $2.2 \pm 0.4$ & $2.1 \pm 0.2$ \\
\hline
\end{tabular}

Legend: ${ }^{\star}$ - based on 60 measurements in each group of cells in 3 untreated and 3 treated patients with cytostatic therapy, ${ }^{\bullet}-$ in arbitrary units, $\boldsymbol{\Delta}$ - in $\mu$ m, \# - significant difference from immature lymphocytes using the t-test $(\mathrm{p}<0.05)$, Th - therapy, NoB - nucleolar body largest axis, PeriNoCh - perinucleolar heterochromatin, PeriNoChB - perinucleolar heterochromatin body

of nucleolar or perinucleolar components using Microsoft Power Point Program (Microsoft, USA)

\section{Results}

Nucleolar bodies and the perinucleolar heterochromatin shell. In specimens stained for DNA, nucleolar bodies of lymphocytes appeared as light unstained areas surrounded by a stained perinucleolar heterochromatin shell that was more distinct in mature cells (Fig. 1, 4). However, it should be mentioned that occasionally it was very difficult or not possible to distinguish nucleolar bodies from light and unstained interchromatin areas. It should be also added that, regardless of the specimen preparation for the light or electron microscopy, the perinucleolar heterochromatin shell and bodies were frequently connected with surrounding condensed chromatin structures in central as well peripheral nuclear regions (Fig. 3, 4, 6).

In specimens stained for RNA, nucleolar bodies were intensely stained and were surrounded by a distinct, but unstained perinucleolar heterochromatin shell that was very distinct in mature small cells (Fig. 2, 5). In addition, nucleolar bodies exhibited a characteristic distribution of RNA [18]. Large nucleoli, mostly present in immature lymphocytes, were characterized by a less or more uniform distribution of RNA. Smaller nucleolar bodies in most of mature lymphocytes usually possessed RNA in the periphery forming a characteristic ring-like periphery (Fig. 2). In pre-apoptotic or apoptotic cells characterized by a heavy chromatin condensation unstained for RNA, nucleolar bodies were associated with unstained and large perinucleolar heterochromatin (Fig. 5).

Largest axis (largest diameter) of nucleolar bodies, perinucleolar heterochromatin shell and perinucleolar DNA image heterochromatin condensation state in immature as well as mature lymphocytes (Table 1 and 2). In general, in immature lymphocytes, the diameter of nucleolar bodies with the surrounding perinucleolar chromatin shell was larger in specimens stained for DNA than after staining for RNA due to the different preparation procedures. In mature lymphocytes the diameter of nucleolar bodies surrounded by the perinuclelar was smaller in both specimens stained for DNA or RNA but still larger after DNA staining than in specimens stained for RNA. Similarly, the diameter of nucleolar bodies - without the perinucleolar chromatin - in immature lymphocytes was larger in specimens stained for DNA than for RNA. In mature lymphocytes the diameter of nucleolar bodies markedly decreased but still was larger after DNA than RNA staining. Thus, as it was expected, the diameter of nucleolar bodies with as well as without the perinucleolar heterochromatin shell in mature lymphocytes was significantly reduced. At this occasion it should be noted that the differences between untreated and treated patients were not significant.

The width of the perinucleolar heterochromatin shell in both immature and mature lymphocytes was larger in specimens stained for DNA than RNA. In mature lymphocytes stained for DNA the increase of the perinucleolar heterochromatin shell was not significant. However, the increase of the perinucleolar unstained heterochromatin shell in mature lymphocytes after staining for RNA was significant. At his occasion it should be noted that the only significant difference of the perinucleolar heterochromatin shell between untreated and treated patients with cytostatic therapy was noted in mature lymphocytes stained for RNA. In treated patients the width of the perinucleolar heterochromatin shell was significantly larger than in those who were untreated at the time of taking samples for the present study,

The heterochromatin condensation expressed by its DNA image optical density measured at the single cell level appeared

Table 2. The unstained perinucleolar heterochromatin width in immature and mature leukemic lymphocytes stained for RNA (mean and standard deviation in $\mu \mathrm{m})^{*}$

\begin{tabular}{lcccc}
\hline Lymphocytes & Th & NoB & NoB+PeriNoCh & $\begin{array}{c}\text { PeriNoCh } \\
\text { width }\end{array}$ \\
\hline immature & 0 & $2.3 \pm 0.2$ & $3.0 \pm 0.1$ & $0.7 \pm 0.1$ \\
mature & & $1.4 \pm 0.2^{*}$ & $2.5 \pm 0.1^{*}$ & $1.1 \pm 0.1^{*}$ \\
immature & + & $2.2 \pm 0.3$ & $3.0 \pm 0.4$ & $0.8 \pm 0.1$ \\
mature & & $1.1 \pm 0.1^{*}$ & $2.6 \pm 0.2$ & $1.5 \pm 0.1^{\#}$ \\
\hline
\end{tabular}

Legend: ${ }^{*}$ - based on at least 100 measurements in each group of cells in 3 untreated and 3 treated patients with cytostatic therapy, ${ }^{\$-}$ significantly different from mature lymphocytes of untreated patients using the $t$-test $(\mathrm{p}<0.02)$ For other legend see Table 1 
to be increased in mature cells in both untreated and treated patients with the chemotherapy. However, the difference was not significant (Table 1).

Perinucleolar heterochromatin bodies (Table 2). It seemed to be interesting that immature as well as mature lymphocytes possessed $1-3$ small perinucleolar heterochromatin bodies of a variable shape which were adjacent to the nucleolus and were easily seen in specimens stained for DNA. In some specimens they were apparently a regular part of the perinucleolar heterochromatin shell (Fig. 3, 4). The image processing, i.e. the contrast increase and background bleaching indicated that perinucleolar heterochromatin bodies were characterized by a high chromatin DNA condensation similarly as the perinucleolar heterochromatin shell (Fig. 3, 4). In patients untreated as well as treated by the cytostatic therapy. The mean diameter of these bodies was slightly larger in mature lymphocytes, but the difference was not significant in comparison with immature cells. It must be also mentioned that the small differences between the diameter of perinucleolar chromatin bodies in lymphocytes of both untreated and treated patients by the cytostatic therapy was also not statistically significant.

Perinucleolar heterochromatin bodies - if present in ultrathin sections - seen by the electron microscope appeared to be a part of the perinucleolar chromatin shell (Fig. 6). They were prominent especially in specimens after the partial or complete removal of the nucleolar RNA containing components (Fig. 6). At larger magnifications all perinucleolar heterochromatin structures such as perinucleolar heterochromatin bodies or perinucleolar heterochromatin shell appeared to be composed of chromatin fibrils similar to those in the heterochromatin within the nucleolar body (Fig, 7).

\section{Discussion}

The present study provided complementary information on the perinucleolar region during the cell maturation using leukemic lymphocytes as a convenient model. Similarly as other nucleolar components, the morphological organization of the condensed perinucleolar heterochromatin was generally related to the lymphocyte maturation. During that process accompanied by a marked decrease of the nucleolar size [7, present results] the width of the perinucleolar heterochromatin shell increased. Such observation is in harmony with the classical hematological cytology that presumed that nucleoli in maturing blood cells are replaced and masked by the "postnucleolar chromatin clumps" [19]. At present, it is already generally known that during the lymphocyte maturation nucleolar biosynthetic activities and the rRNA transcription are markedly decreased. On the other hand, the function of the perinucleolar chromatin region is less clarified although it seems to be likely that it participates in other nucleolar activities including the transcription of other RNA species than the rRNA $[1,2,4]$. Thus the enlarged width of the perinucleolar heterochromatin, i.e. perinucleolar heterochromatin enlarge- ment might be related to the gene silencing in that nuclear region during the cell maturation. The increasing chromatin condensation and heterochromatin formation are generally considered to reflect the gene silencing at present [1,20-24], Such speculation would be also in harmony with the larger perinucleolar heterochromatin region in not-proliferating hepatocytes in comparison with proliferating malignant hepatoma cells [25]. Similarly, another electron microscopic observation on not-leukemic T lymphocytes demonstrated that perinucleolar condensed chromatin regions were apparently larger in resting than in proliferating cells stimulated with phytohemaglutinin [26].

The presence of one or several heterochromatin bodies in the perinucleolar region in leukemic B-lymphocytes seems to be interesting. Since perinucleolar heterochromatin bodies were present in both immature and mature leukemic lymphocytes regardless of the therapy, they appeared to be regular components of the perinucleolar nuclear region in these leukemic cells. In would be very difficult to speculate about their function on the base of the present cytochemical observation and more studies and different methodical approaches are required in this direction. However, the perinucleolar region represents a special nuclear compartment that is more expressed in malignant cells and possibly is associated with a special DNA locus $[2,4,5]$. Thus, the presence of perinucleolar heterochromatin bodies in the perinucleolar region of leukemic lymphocytes is not contradictory with the present knowledge on that nuclear territory. It should be also noted that perinucleolar heterochromatin bodies seen by both light as well electron microscopy including DNA and RNA cytochemistry differed from other nuclear bodies present at the nucleolus, In contrary to other known nuclear bodies $[2,3,20]$, perinucleolar heterochromatin bodies in studied leukemic lymphocytes consisted only of DNA containing fibrils and did not contain other structural components including RNA.

Acknowledgements: The present study was supported in part by the Czech Ministry of Health project for the research organization development. The authors would like to express their gratitude to the physicians of the Clinical section of the Institute and General Medical Hospital in Prague.

\section{References}

[1] PEDERSON T. The plurifunctional nucleolus. Nucleic Acid Res 1998; 26: 3871-3876. http://dx.doi.org/10.1093/nar/ $\underline{26.17 .3871}$

[2] POLLOCKG, HUANG B. The perinucleolar compartment. J Cell Biochem 2000; 15: 189-183.

[3] HUANG S. Review: perinucleolar structures. J Struct Biol 2000;129: 233-240. http://dx.doi.org/10.1006/jsbi.2000.4247

[4] SLUDARCZYK A, KAMATH R, WANG C, ANCHEL D, POLLOCK $C$ et al. Structure and function of the perinucleolar compartment in cancer cells. Cold Spring Harb Symp 
Quant Biol 2010; 75: 599-605. http://dx.doi.org/10.1101/ sqb.2010.75.026

[5] NORTON JT, WANG Ch, GJIDODA A, HENRY W., HUANG $\mathrm{S}$. The perinuclelar compartment is directly associated with DNA. J Biol Chem 2009: 284, 4090-410.

[6] SMETANA K, BUSCH H. The nucleolus and nucleolar DNA, In The cell nucleus 1 . Busch H. ed. New York:Academic Press, 1974: 75-148. http://dx.doi.org/10.1016/B978-0-12-1476014.50010-5

[7] SMETANA K, KARBAN J, TRNENY M, To the nucleolar bodies (nucleoli) in cells of the lymphocytic lineage in patients suffering from B-chronic lymphocytic leukemia. Neoplasma 2010; 57: 495-500. http://dx.doi.org/10.4149/neo $2010 \quad 06 \quad 495$

[8] SMETANA K, KARBAN J, TRNENY M. Heterochromatin condensation in central and peripheral nucleolar regions of maturing lymphocytes in he peripheral blood of patients suffering from B chronic lymphocytic leukemia - a cytochemical study. Neoplasma 2011; 58: 476-481. http://dx.doi. org/10.4149/neo 201106476

[9] KLOBUSICKA M, KUSENDA J, STEVULOVA I, KOVARIKOVA A, BABUSIKOVA O. Possible prognostic value of nucleolar morphology in pathologic cells of B-chronic lymphocytic leukemia. Neoplasma 2010; 57: 429-437. http:// dx.doi.org/10.4149/neo $2010 \quad 05 \quad 429$

[10] SMETANA K, LEJNAR J, POTMESIL M. A note to the demonstration of DNA in nuclei of blood cells in smear preparation. Folia Haematol 1967; 88: 305-317.

[11] BUSCH H, SMETANA K, The nucleolus. New York: Academic Press, 1970.

[12] SMETANA K, POTMESIL M, LEJNAR J. A further contribution to the demonstration of RNA and nucleoli in blood cells in smear preparations. Folia Haematol 1969; 91: 381-394.

[13] OCHS RL. Methods used to study structure and function of the nucleolus. Methods Cell Biol 1998; 53: 303-321.

[14] POLITI EN, LAZARIS AC, KAVANTZAS N, KOUTELINI $\mathrm{H}$. Comparison between morphometry and immunostaining of malignant in non-small cell lung cancer. Anal Quant Cytol Histol 2003; 25: 162-176.
[15] TSELENI S, KAVANTZAS N, VOVA D, ALEXANDRATOUR E, IOANNOU-LAMBROULI M. et al. Findings of computerized nuclear morphometry of papillary thyroid carcinoma in correlation with the age of the patients. Gen Diagn Pathol 1997; 143: 23-27.

[16] SMETANA K. Electron microscopy of lymphocytes. Methods in cancer research 1970; 5: 455-478.

[17] MOLLENHAUER HH. Plastic embedding mixtures for use in electron microscopy. Stain technol 1964; 39: 111-114.

[18] SMETANA K. The nucleolus through the years. Editorial. J Appl Biomed 2011; 9: 119-127. http://dx.doi.org/10.2478/ v10136-011-0010-7

[19] UNDRITZ E. Hämatologische tafeln. Sandoz 1972, Basel.

[20] SPECTOR DL, Macromolecular domains within the cell nucleus. Ann Rev Cell Biol 1993; 9: 265-315. http://dx.doi. org/10.1146/annurev.cb.09.110193.001405

[21] FRENSTER JH, Ultrastructure and function of heterochromatin and euchromatin. In The cell nucleus, Busch H ed, New York,:Academic Press, 1974, 565-581 http://dx.doi. org/10.1016/B978-0-12-147601-4.50020-8

[22] ZHIMULEV IF, BELIAEVA ES, Heterochromatin, gene position effect and gene silencing. Genetika 2003; 39: 187-201.

[23] GRIGORYEV SA, BULYNKO VA, POPOVA EY. The end adjusts the means: heterochromatin remodeling during terminal cell differentiation. Chromosome Res 2006; 14: 53-69. http://dx.doi.org/10.1007/s10577-005-1021-6

[24] GREVAI SI, JIVA S. Heterochromatin revisited. Nature Reviews Genetics 2007; 8: 35-49. http://dx.doi.org/10.1038/ $\underline{\operatorname{nrg} 2008}$

[25] UNUMA T, SMETANA K, BUSCH H, A morphologic study on the chromatin areas associated with the nucleolus. Exp Cell Res 1967; 48: 665-671. http://dx.doi.org/10.1016/00144827(67)90340-0

[26] RASKA I, RYCHTER Z, SMETANA K, Fibrillar centers and condensed nucleolar chromatin in resting and stimulated human lymphocytes, Z mikrosk anat Forsch Leipzig 1983; 97: 15-32. 